\title{
Ergonomic Design and Evaluation of a Pilot Oxygen Mask for Korea Air Force Pilots
}

\author{
Wonsup LEE ${ }^{1}$, Daehan JUNG ${ }^{2}$, Seikwon PARK ${ }^{3}$, Hee-Eun $\mathrm{KIM}^{4}$, Heecheon YOU ${ }^{\star 1}$ \\ ${ }^{1}$ Industrial and Management Engineering, POSTECH, South Korea; \\ ${ }^{2}$ Department of Mechanical Engineering, Korea Air Force Academy, Cheongwon, South Korea; \\ ${ }^{3}$ Department of Systems Engineering, Korea Air Force Academy, Cheongwon, South Korea; \\ ${ }^{4}$ Department of Clothing and Textiles, Kyungpook National University, Daegu, South Korea
}

http://dx.doi.org/10.15221/14.084

\begin{abstract}
The present study developed a virtual fit analysis (VFA) method to design an oxygen mask which fits Korean Air Force (KAF) pilots. The VFA method used 3D face scan data of $336 \mathrm{KAF}$ pilots to find the most proper shape of an oxygen mask for KAF pilots. The oxygen mask design revised in the study showed a $27 \%$ design improvement effect on average in terms of fit evaluated by the VFA method. Additionally, this study evaluated the revised oxygen mask prototypes with $88 \mathrm{KAF}$ pilots to experimentally verify the design improvement effect in terms of discomfort, pressure, and suitability for military equipment (slippage and stability in flight-like situations). The discomfort of the revised mask was $33 \sim 56 \%$ lower on average than the existing oxygen mask. In terms of the pressure, the revised mask showed $11 \sim 33 \%$ of improvement on average compared to the existing mask. Furthermore, on high gravity situation, the slippage distance of the revised mask was $24 \%$ shorter on average than the existing mask. The proposed VFA method can be applied to the design and evaluation of wearable products that require an ergonomically better fit for a target population.
\end{abstract}

Keywords: pilot oxygen mask, mask design, mask evaluation, virtual fit analysis method, 3D face scan images

\section{Introduction}

An oxygen mask is required to be designed to properly fit the face of a target population to prevent users from the harmful atmosphere. The MBU-20/P (Gentex Corporation, U.S.A.) pilot oxygen mask, frequently used for the Korean Air Force (KAF) pilots, was originally designed using face anthropometric data of 2420 U.S. Air Force (USAF) personnel collected by Churchill et al. [1] and had been improved by applying the three-dimensional face scan data of 60 ( 30 males and 30 females) pilots [2]. A survey conducted by Lee et al. [3, 4] on the usability evaluation of the MBU-20/P mask identified that more than 60 percent of KAF pilots suffered from excessive pressure and/or oxygen leakage around the nasal root due to the lack of fit from the oxygen mask to the face, which is most likely caused by a significant difference in facial shape and size between KAF pilots and USAF personnel [5].

Previous research on a mask design used representative face models; however, those masks didn't consider the facial characteristics analyzed to a significant amount of a target population in their research. Yatapanage and Post [6], Gross et al. [2], Han and Choi [7], Han et al. [8], and Song and Yang [9] proposed mask design methods based on an average face size or $1 \sim 3$ of representative face models. However, the previous study didn't explain details of the mask design method based on the 3D facial data. Also, there were a lack of systematical consideration for whether the proposed representative face models could significantly represent a target population.

Evaluation of an appropriate pressure to a mask is required to design for better fit to the mask users; however, there is no study has introduced a systematic approach for pressure evaluation. An appropriate range of fit is important to avoid discomfort due to excessive pressure or oxygen leakage due to lack of fit [9]. An experimental method for a pressure evaluation is required to understand mask fit characteristics for a mask design which can provide better fit and comfort to users. To evaluate the mask pressure, [10] applied Tactilus freeform sensor system (Sensor Products Inc., Madison, NJ, USA) on six facial points (nasal bridge, top of right cheek, top of left cheek, bottom of right cheek, bottom of left cheek, and chin) of a mannequin head. However, there are no researches which measure mask pressure by facial areas with mask users.

The present study developed an oxygen mask design method by using a virtual fit analysis and applied it to the design of an oxygen mask which fits KAF pilots. The oxygen mask appropriate to

* hcyou@postech.edu; +82-54-279-2210; http://edt.postech.ac.kr 
the facial characteristics of KAF pilots was designed by using 3D face scan data of all $336 \mathrm{KAF}$ pilots. Prototypes of the designed oxygen mask were ergonomically evaluated by $83 \mathrm{KAF}$ pilots in terms of discomfort, pressure, and suitability for military equipment.

\section{Oxygen Mask Design Method}

\subsection{Virtual Fit Analysis (VFA) Method}

A virtual fit analysis (VFA) is a method which automatically fits the oxygen mask CAD on the 3D facial scan images and virtually evaluates mask wearing characteristics (e.g., fit, clearance). A VFA system coded by Matlab 2008a (The MathWorks, Inc., U.S.A.) was used to automatically align the 3D face scan images and the oxygen mask CAD and quantitatively analyze their fit [11]. The actual oxygen mask made of silicone rubber is deformed when a pilot wears the mask; however, the oxygen mask CAD infiltrates into the 3D face during virtual fitting as highlighted in red in Figure 1. Therefore, this study identified the fit as an infiltrated distance of the oxygen mask CAD into the 3D face image. Deep infiltration (e.g., infiltrated distance $>10 \mathrm{~mm}$ ) means an excessive pressure, while no infiltration (infiltrated distance $\leq 0 \mathrm{~mm}$ ) can be explained as an oxygen leakage. The fit is analyzed by $1 \mathrm{~mm}$ according to the vertical location of the face (see Figure 2). For instance, the oxygen mask is slightly fitted or not fitted at the nasal root area (vertical location: between 0 and $10 \mathrm{~mm}$ ), and deeply fitted ( maximum infiltrated distance $=20 \mathrm{~mm}$ ) at the nasal side and zygomatic bone areas (vertical location: between 10 to $60 \mathrm{~mm}$ ).
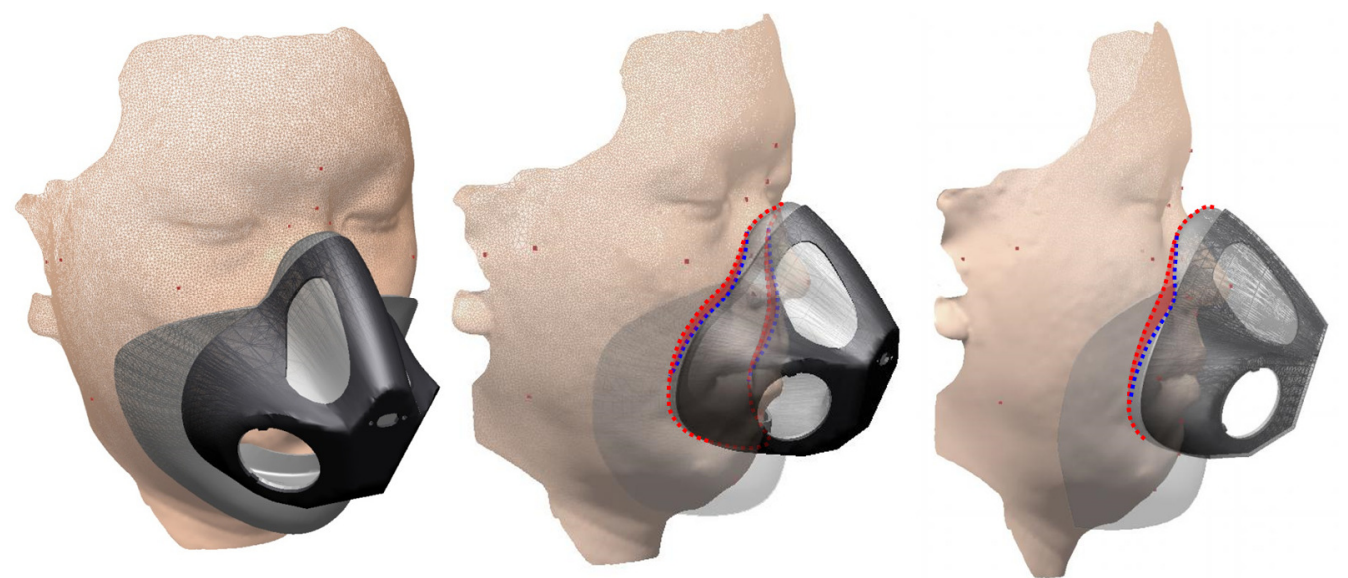

Fig. 1. Illustration of a virtual fit analysis of an oxygen mask design on a pilot's face

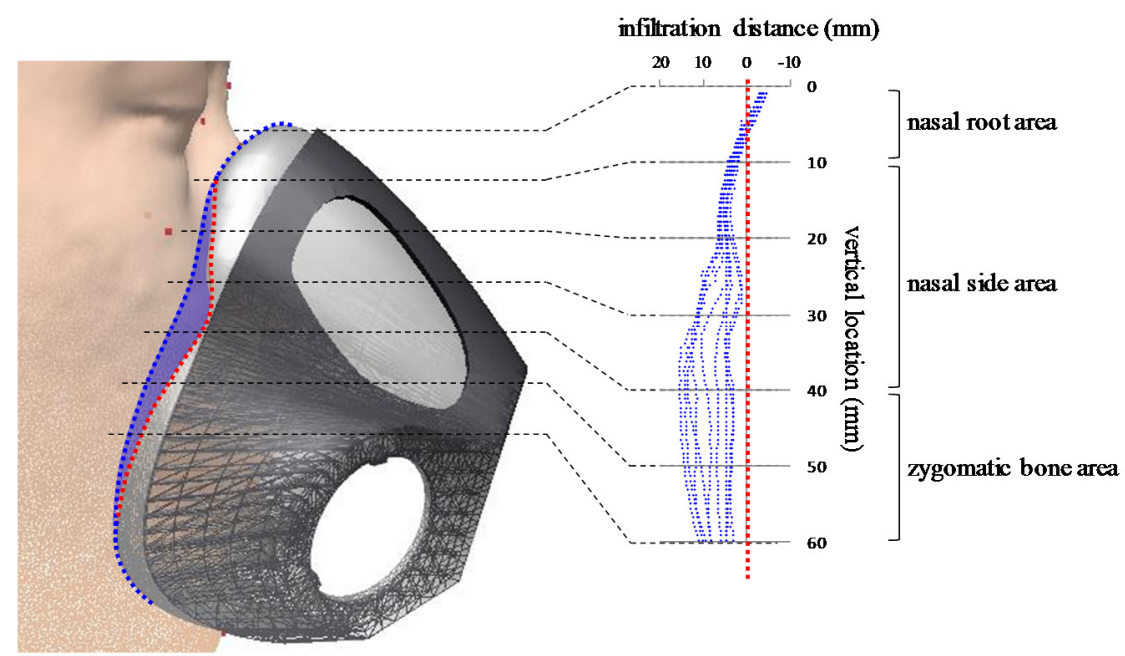

Fig. 2. Fit of the oxygen mask according to vertical location

\subsection{Oxygen Mask Design Based on the VFA Method}

This study designed the oxygen mask shapes (four sizes: small narrow, medium narrow, medium wide, and large wide) which were highly appropriate to the 3D facial data of $336 \mathrm{KAF}$ pilots. The oxygen mask shape was revised through an iterative process between a design update using Rhino 3D 
(McNeel, U.S.A.), CAD software and an evaluation of its fit using the VFA system. The 3D faces of 336 KAF pilots were used to design the oxygen masks for KAF pilots. The sample size of KAF pilots was statistically identified based on ISO 15535 by Lee et al. [3]. For an example of the medium narrow size $(n=113)$, the existing oxygen mask showed less or no pressure (infiltrated distance $<0 \mathrm{~mm}$ ) on the nasal root area (see red circles in Figure 3.a), and an excessive pressure (infiltrated distance $>10 \mathrm{~mm}$ ) on the nasal side and zygomatic bone area, while the revised oxygen mask showed moderate fit at all locations on the face area (see Figure 3.b).

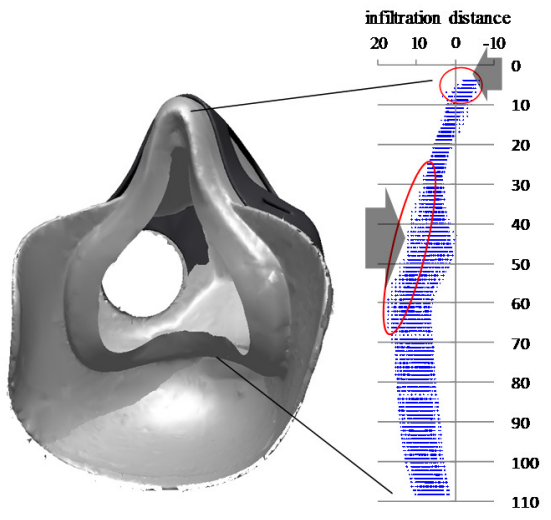

(a) Existing oxygen mask

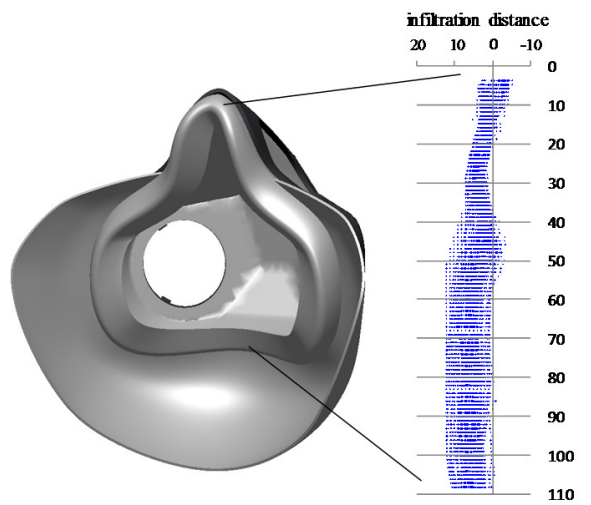

(b) Revised oxygen mask

Fig. 3. Fit analysis for the existing and revised oxygen mask

\subsection{Evaluation of Oxygen Mask Shapes through the VFA Method}

The revised oxygen mask showed an average $27 \%$ improvement in terms of design satisfaction ratio by facial area. The existing mask satisfied an average $55.3 \%(21 \sim 89 \%$ by facial area) of the sample population in terms of a fit criteria (indicated by red lines in Figure 4) identified by this study, while the revised mask satisfied $82.3 \%$ (66 92\% by facial area) on average (Figure 4).

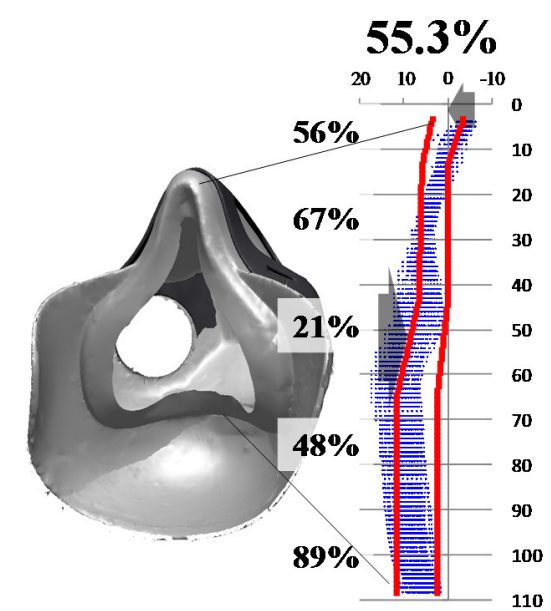

(a) Existing oxygen mask

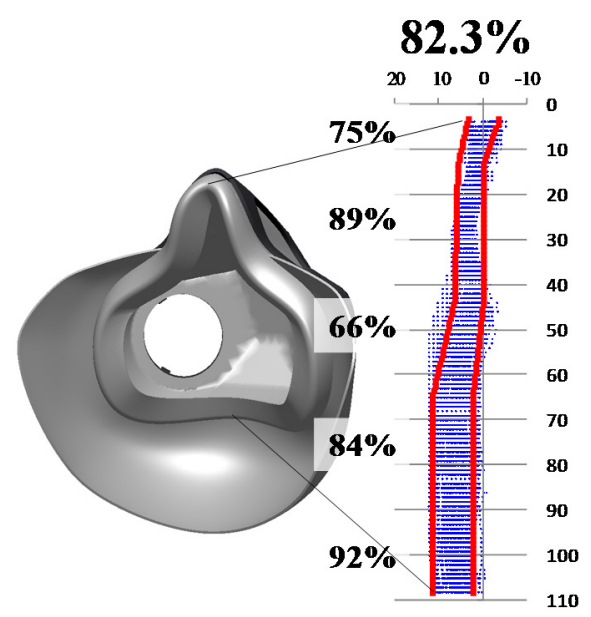

(b) Revised oxygen mask

Fig. 4. Evaluation of design satisfaction through virtual fit analysis method $(n=336)$

\section{Oxygen Mask Evaluation Method}

\subsection{Participants}

An ergonomic usability evaluation between the existing and revised oxygen masks was conducted with $83 \mathrm{KAF}$ pilots (81 males and 2 females; age: $25 \sim 43$ years old) who currently use the MBU-20/P oxygen mask. An additional $5 \mathrm{KAF}$ male pilots participated for evaluation of suitability of the revised mask in high-G and low atmospheric pressure situations. The evaluation was conducted at three KAF bases, the KAF Academy, and the Aerospace Medical Center of KAF. 


\subsection{Apparatus}

This study manufactured prototypes of the revised oxygen masks by sizes (SN, MN, MW, and LW). To evaluate the oxygen masks under similar conditions, this study used similar materials as the MBU-20/P for the prototype of the revised oxygen mask (facepiece and hardshell). Material properties (e.g., hardness, toughness, tensile, and elasticity) were determined by a panel of materials experts. The existing components (e.g., valves, straps, and microphone) were used with the revised facepiece and hardshell.

Hypobaric aviation physiology training chamber (Environmental Tectonics Corporation, USA) and a high-G training equipment (Environmental Tectonics Corporation, USA) were used for the evaluation of suitability for military equipment. The aviation physiology training chamber and the high-G training equipment at the Aerospace Medical Center are part of a regular training facility for the KAF pilots. The aviation physiology training chamber (Figure 5.2b) can simulate various atmospheric pressure corresponding altitude $(0 \sim \geq 25,000 \mathrm{ft}$.), and the chamber supplies three types of air according to altitude $(<25,000 \mathrm{ft}$.: supplement of air with $20 \%$ oxygen, $\geq 25,000 \mathrm{ft}$.: supplement of $100 \%$ oxygen, emergency mode: excessive supplement of 100\% oxygen at any altitude). This study evaluated the stability of the revised oxygen mask according to the various types of air supply. Also, the pilot oxygen mask is used for a stable supply of oxygen to the pilot while a mission is conducted at high altitude where oxygen is lacking and in high gravity acceleration where the oxygen mask can slip to downward on the face. Therefore, the prototype of the revised oxygen mask was required to be tested in flight-like environments which simulate lack of oxygen and high-G.

\subsection{Evaluation Protocol}

A usability evaluation was conducted in terms of discomfort and pressure through a five-step protocol (see Figure 5). Pilots used their own MBU-20/P mask to evaluate the existing oxygen mask. For the revised mask evaluation, they selected one of prototypes among four sizes considering fit to his/her face shape and size. Pilots wore the existing or revised mask for 10 minutes respectively, then evaluated discomfort and pressure of the mask. The evaluation order of the existing and revised oxygen mask was counterbalanced.

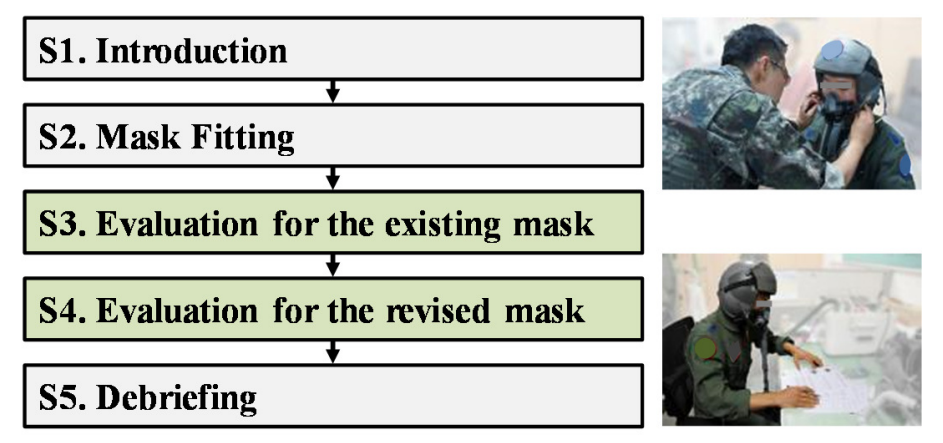

Fig. 5. Oxygen mask evaluation protocol

The discomfort of the existing and revised oxygen masks were evaluated for six facial areas (nasal root, nasal side, zygomatic bone, cheek, bottom lip, and chin) by a 7-point scale (0: no discomfort, 1: rarely uncomfortable, 2: somewhat uncomfortable, 3: slightly uncomfortable, 4: moderately uncomfortable, 5: quite uncomfortable, 6: very uncomfortable, and 7: extremely uncomfortable) using a questionnaire developed by this study (see Figure 6).

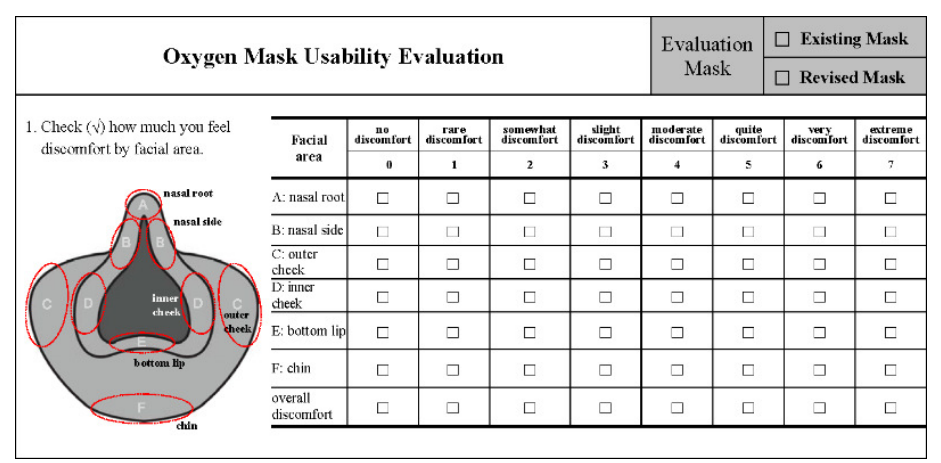

Fig. 6. Subjective evaluation questionnaire (illustration) 
The pressure of the existing and revised oxygen masks were measured by Prescale pressure indicating film (Fujifilm, Japan) and analyzed by a pressure analysis system developed by this study. The pressure of the existing and the revised oxygen masks was measured by the pressure film, then evaluated by a pressure analysis system developed in this study. The pressure evaluation was conducted by a five-step process: (1) preparation of pressure film, (2) measurement of pressure, (3) scanning of pressure film, (4) refinement of scan image using Photoshop, and (5) analysis of pressure through pressure analysis system coded using MATLAB (see Figure 7). The film shows amount of pressure between the oxygen mask and face, and is represented by a darkness (white: no pressure; black: maximum pressure) according to amount of pressure. After scanning the film (image size: $220 \times 220$ pixels), the system analyzes the amount of pressure (0: no pressure; 100: maximum pressure) on four facial areas (nasal root, nasal side, cheek, and bottom lip) (see Figure 8). An average was used for an analysis of the pressure (a mean pressure value at each facial area); also, a pressed area (pressure $\geq 40$; unit: number of pixels) and an excessively pressed area (pressure $\geq 70$; unit: number of pixels) were defined by this study.

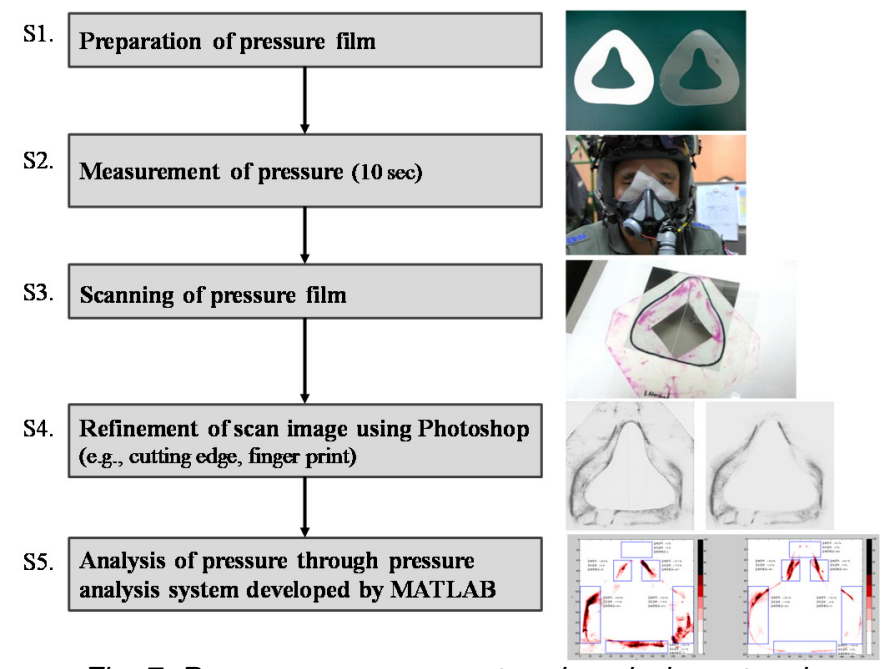

Fig. 7. Pressure measurement and analysis protocol

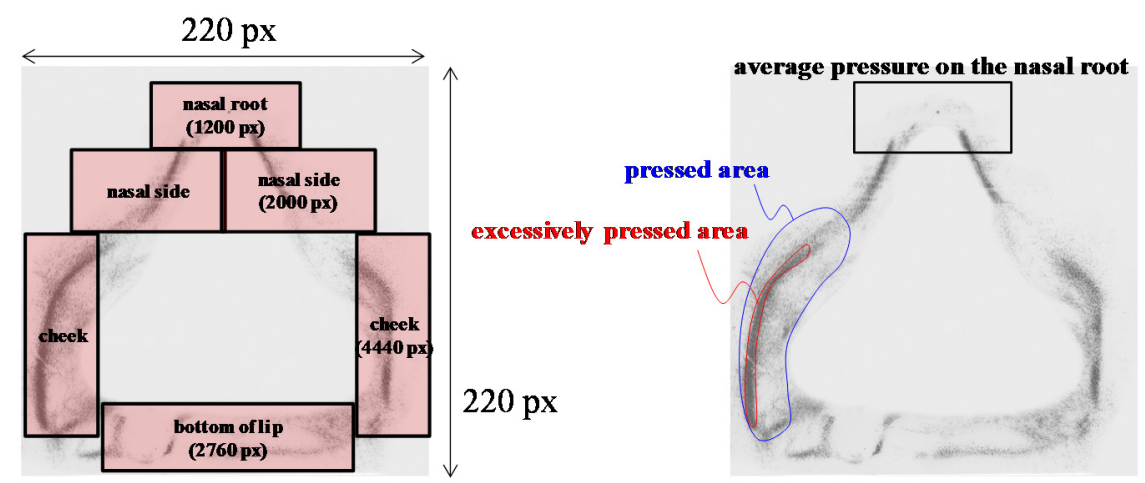

Fig. 8. Example of pressure analysis

Separately from the usability evaluation, the revised mask's suitability for military equipment was evaluated in situations of high gravity acceleration and low atmospheric pressure with 5 pilots. The evaluation of low atmospheric pressure was conducted by using an aviation physiology training chamber which supplies various types of air (e.g., atmospheric pressure at <25,000 feet situation: supplement of air with $20 \%$ oxygen, atmospheric pressure at $\geq 25,000$ feet situation: supplement of $100 \%$ oxygen) to the pilot through the oxygen mask (see Figure 9.a). Pilots subjectively evaluated the stability of the revised oxygen mask according to the various types of air supply. Also, the evaluation of high gravity acceleration (9G) was conducted by using a high-G training equipment to identify a slippage of the oxygen mask at the face (see Figure 9.b). The slippage of the mask was evaluated by questionnaire (0: no discomfort, 7: extremely uncomfortable due to slippage) and video inspection. 


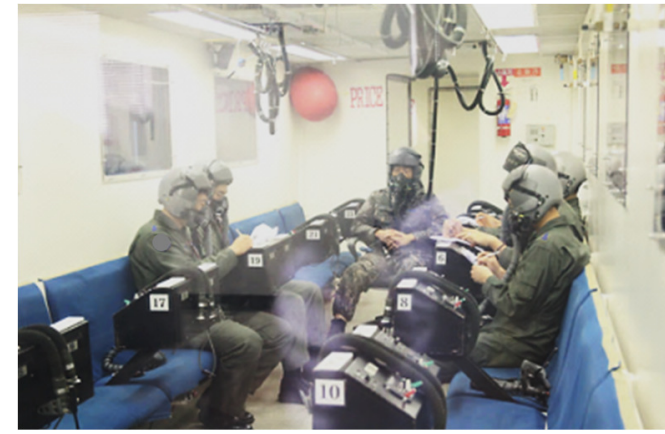

(b) Evaluation in low atmospheric pressure

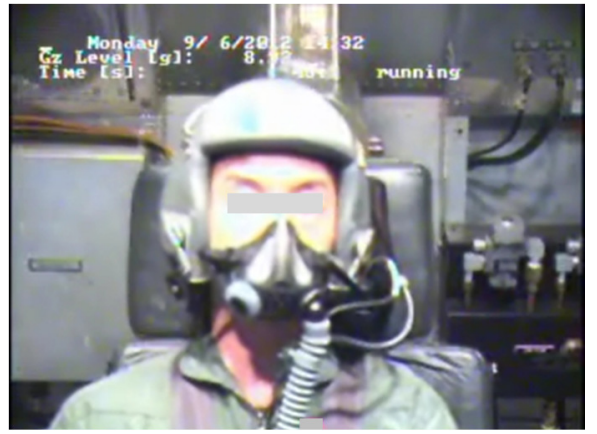

(a) Evaluation in high gravity

Fig. 9. Evaluation of suitability for military equipment

\section{Results}

\subsection{Subjective Evaluation}

The KAF pilots preferred the revised oxygen mask over the existing mask in terms of comfort. The discomfort of the revised mask was $33 \sim 56 \%$ lower on average than the existing mask by facial areas. Specifically, this study selected 43 pilots who felt the high discomfort (discomfort score $>3$ ) at nose (nasal root or nasal side area) in the existing mask, and found that the discomfort of the revised mask among them was $56 \sim 81 \%$ lower on average than the existing mask by facial areas (see Figure 10.b). Additionally, $73 \%$ of pilots ( $n=61$ ) chose the revised mask as the one they preferred for own face (see Figure 11).

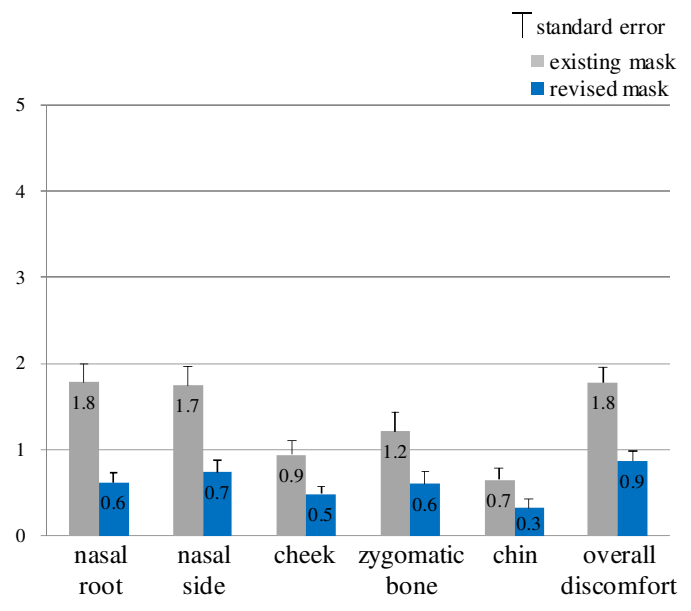

(a) Results of all 83 pilots

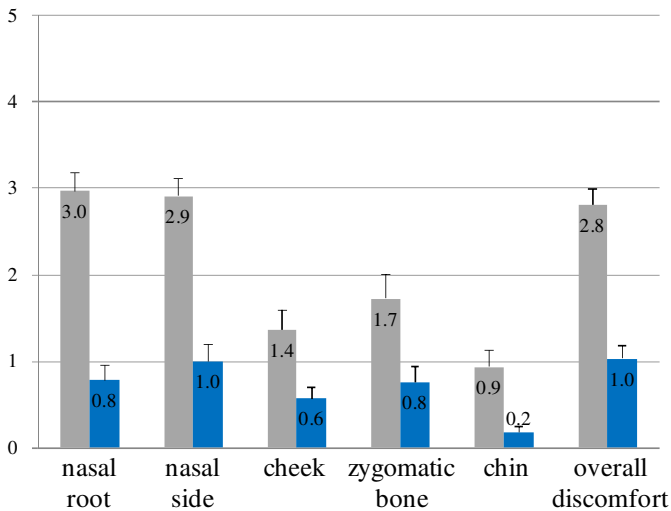

(b) Results of 43 pilots who felt high discomfort at the nose

Fig. 10. Discomfort analysis results

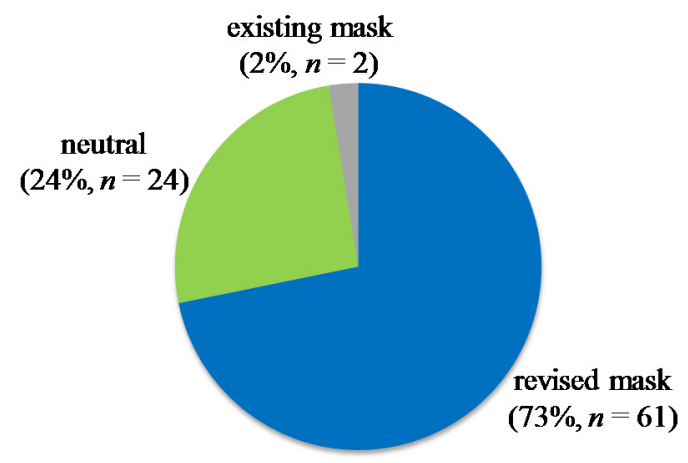

Fig. 11. Preference results $(n=87)$ 


\subsection{Pressure Evaluation}

The revised mask showed less pressure than the existing mask, and evenly fitted to the pilots' face (see Figure 12). The average pressure of the revised mask was $11 \sim 25 \%$ lower on average than the existing mask by facial areas (see Figure 13.a) except the bottom lip. And the pressed area of the revised mask was $24 \sim 33 \%$ lower on average than the existing mask by facial areas (see Figure 13.b) except the bottom lip. In terms of the bottom lip area, the average pressure and the pressed area of the revised mask were $14 \%$ and $23 \%$ higher on average than those of the existing mask. However, this can be interpreted as a better fit instead of excessive pressure, because the discomfort of the revised mask was lower than the existing mask at the bottom lip area. Lastly, the excessive pressed area of the revised mask was $8 \sim 40 \%$ lower on average than the existing mask by facial areas (see Figure 13.c).

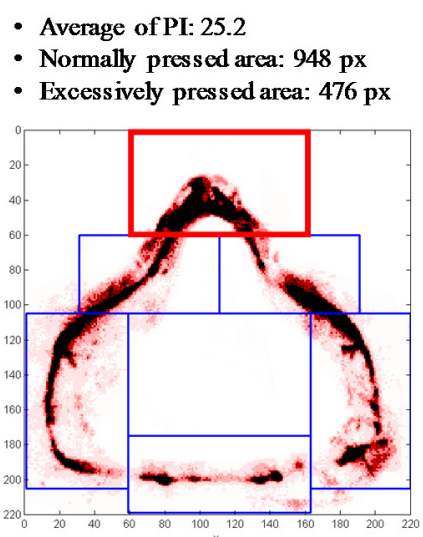

Existing mask (size: small narrow)
- Average of PI: $10.0(60 \% \Downarrow)$

- Normally pressed area: $170 \mathrm{px}(82 \% \downarrow)$

- Excessively pressed area: 82 px $(83 \% \downarrow)$

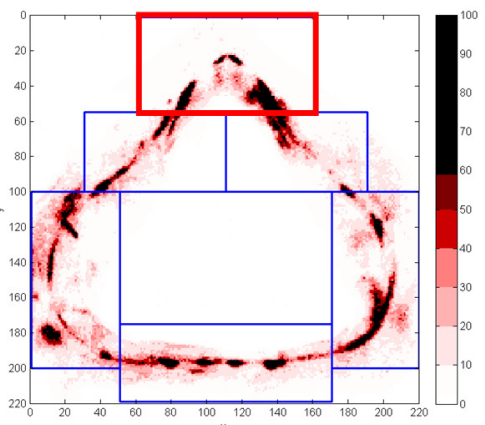

Revised mask (size: medium wide)

Fig. 12. Pressure analysis result at nasal root (illustrated)

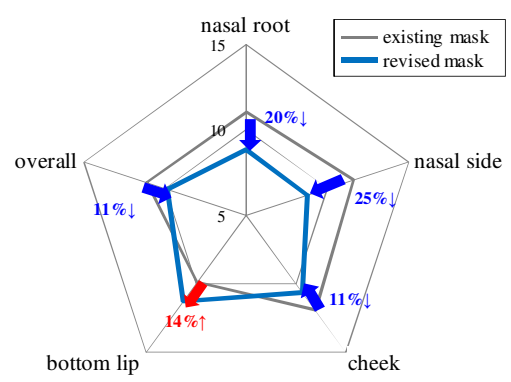

(a) Average of pressure

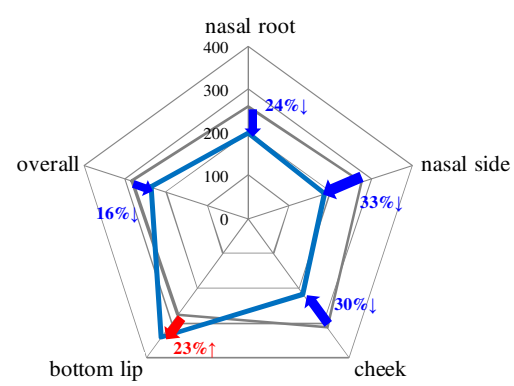

(b) Pressed area

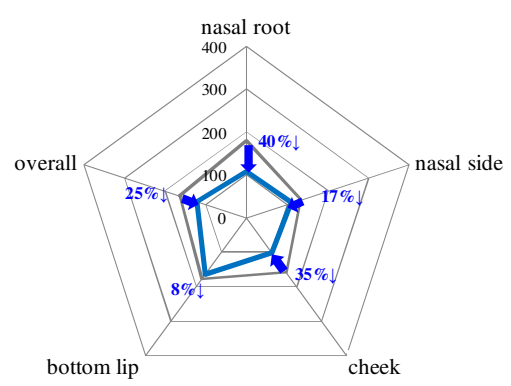

(c) Excessively pressed area

Fig. 13. Pressure analysis results $(n=83)$

\subsection{Suitability Evaluation}

The discomfort due to slippage of the revised mask (score $=0.4$ on average) was $14 \%$ lower on average than the existing mask (score $=2.8$ on average). A slippage distance was measured by video inspection; and the slippage distance of the revised mask was shortened by $24 \%$ on average compared to the existing mask (see Figure 14). And, all five pilots reported that there were no problems with the revised mask in various air supply situations that varied according to atmospheric pressure.

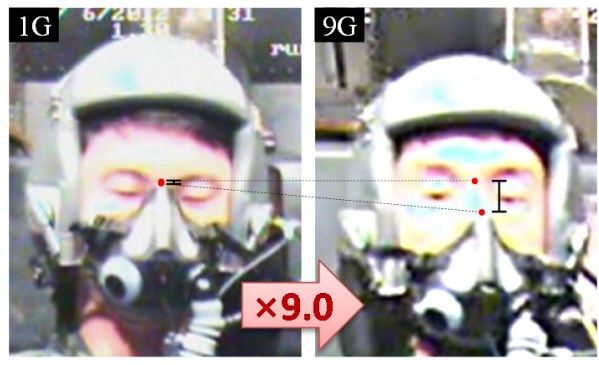

(a) Existing oxygen mask

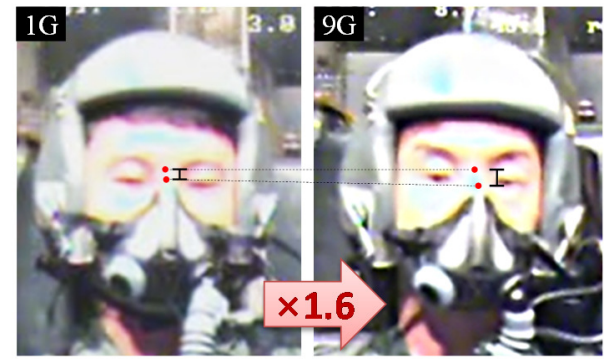

(b) Revised oxygen mask

Fig. 14. Video analysis results (illustrated) 


\section{Discussion}

This study developed the VFA method to design an ergonomic oxygen mask shape which is highly appropriate for KAF pilots (see Figure 15). The previous studies used an average or a few representative faces for a mask design; however, this study considered how the mask would be more effectively applied to the specific target population (KAF pilots). And, the previous study did not verify a representativeness of the face models; this means a mask shape design based on the face models may not be fitted to a large number of a target population. On the other hand, this study could validate the design improvement effect of the revised oxygen mask with the statistically significant number $(n=$ 336) of the target population through the VFA method.
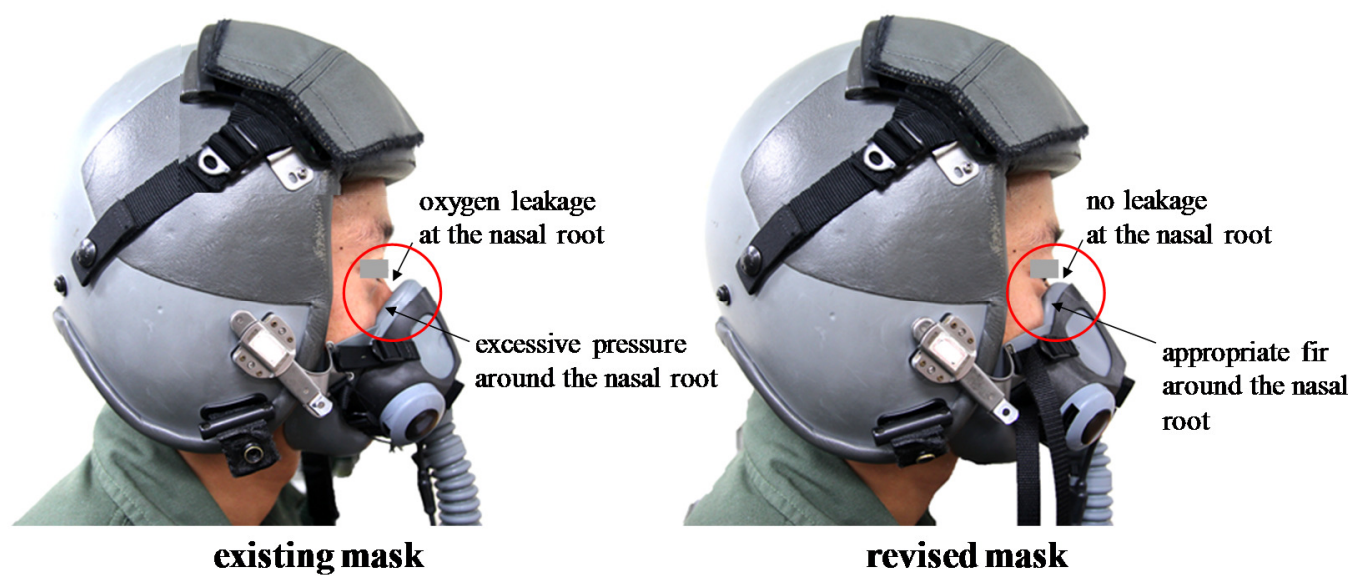

Fig. 15. Comparison of fit between the existing and revised mask designs

This study developed the ergonomic usability evaluation protocol and evaluated the fit of the oxygen mask in both qualitative and quantitative terms. The previous study mainly proposed some experimental methods for identification of air penetration, while this study experimentally measured the pressure between the mask and face by using pressure indicating film. Also, the previous study didn't propose any subjective measures for the mask usability test, while this study developed and applied the questionnaire which can evaluate the fit of the oxygen mask in terms of not only discomfort but also oxygen leakage, slippage, and overall satisfaction. Therefore, this study could experimentally validate the design improvement effects of the revised oxygen mask and the oxygen mask design method simultaneously.

The proposed VFA method is appropriate to design and evaluate ergonomically wearable products in the early stage of the product development process. In the case of the oxygen mask, this study made metal molds for the mask prototypes. However, because of cost, it is very difficult to make numerous molds to find the proper shape of the mask. Therefore, the VFA method proposed by this study can be usefully applied for design and evaluation of the product shape at the stage prior to the prototype manufacturing.

The revised oxygen mask was suitable to use as military equipment due to stable performance in the situations of PBG, low atmospheric pressure, and high-G. Because the pilot oxygen mask is used in extreme environments such as high altitude and high gravity acceleration, the usability evaluation in those situations is necessary to identify the suitability and stability of the design for the pilots. Of course, the fit of the revised oxygen mask was improved, and the slippage of the revised design was decreased in the high-G situation. Also, the revised oxygen mask was evaluated to have no functional problems in PBG and low atmospheric pressure situations. Furthermore, additional in-depth examinations, such as material properties evaluation, compatibility testing, or environmental assessment are required in order to provide the revised oxygen mask to the KAF pilots.

\section{Acknowledgement}

This research was jointly supported by an Air Force Logistics Command grant funded by the Republic of Korea Air Force and the Basic Science Research Program through the National Research Foundation of Korea (NRF) funded by the Ministry of Education, Science, and Technology (2013R1A2A2A04014195). 


\section{References}

[1] E. Churchill, P. Kikta, and T. Churchill, "The AMRL Anthropometric Data Bank Library: Volumes I-V (AMRL-TR-77-1, ADA047314)". Wright-Patterson Air Force Base, OH: Aerospace Medical Research Laboratory, 1977.

[2] M. E. Gross, S. E. Taylor, D. N. Mountjoy, and J. Hoffmeister, "Anthropometric research on the sizing of the MBU-20P (AFRL-HE-WP-TR-2002-0181)". Wright-Patterson Air Force Base, OH: Human Effectiveness Directorate, Crew System Interface Division, 1997.

[3] W. Lee, J. Jeong, J. Park, E. Jeon, H. Kim, D. Jung, S. Park, and H. You, "Analysis of the facial measurements of Korean Air Force pilots for oxygen mask design", Ergonomics, Vol.56, No.9, 2013, pp.1451-1464, http://dx.doi.org/10.1080/00140139.2013.816376.

[4] W. Lee, H. Kim, D. Jung, S. Park, and H. You, "Ergonomic design and evaluation of a pilot oxygen mask", In Proc. of Human Factors and Ergonomics Society $57^{\text {h }}$ Annual Meeting, San Diego, CA, 2013.

[5] W. Lee, J. Park, J. Jung, E. Jeon, H. Kim, S. Park, and H. You, "Analysis of the Facial Anthropometric Data of Korean Pilots for Oxygen Mask Design", In Proc. of the Human Factors and Ergonomics Society 56th Annual Meeting, Boston, MA, USA, 2012.

[6] K. G. Yatapanage and K. Post, "Measurement of 3-D facial contours for the design of half-face respirators", American Industrial Hygiene Association Journal, Vol.53, No.1, 1992, pp.19-26, http://dx.doi.org/10.1080/15298669291359258.

[7] D. Han and K. Choi, "Facial dimensions and predictors of fit for half-mask respirators in Koreans". American Industrial Hygiene Association Journal, Vol.64, No.6, 2003, pp.815-822, http://dx.doi.org/10.1080/15428110308984877.

[8] D. Han, J. Rhi, and J. Lee, "Development of prototypes of half-mask facepieces for Koreans using the 3D digitizing design method: A pilot study", Annals of Occupational Hygiene, Vol.48, No.8, 2004, pp.707-714, http://dx.doi.org/10.1093/annhyg/meh068.

[9] J. C. Dai, J. Z. Yang, and Z. Zhuang, "Sensitivity analysis of important parameters affecting contact pressure between a respirator and a headform", International Journal of Industrial Ergonomics, Vol.41, No.3, 2011, pp.268-279, http://dx.doi.org/10.1016/j.ergon.2011.01.007.

[10]Z. Lei, J. Yang, and Z. Zhuang, "Headform and N95 filtering facepiece respirator interaction: Contact pressure simulation and validation", Journal of Occupational and Environmental Hygiene, Vol.9, 2012, pp.46-58, http://dx.doi.org/10.1080/15459624.2011.635130.

[11]W. Lee, J. Jeong, D. Son, S. Park, D. Jung, H. Kim, M. You, C. Park, and H. You, "Development of a methodology to design a pilot oxygen mask based on virtual fit testing method", In Proc. of the 2012 Spring Conference of the Korean Institute of Industrial Engineers, Gyeongju, South Korea, 2012. 